\title{
ISO Spectroscopy of $\mathbf{H}_{2}$ in Star Forming Regions
}

\author{
By M. E. van den Ancker ${ }^{1,2}$, P. R. Wesselius ${ }^{3}$ \\ AND A. G. G. M. Tielens ${ }^{3,4}$ \\ ${ }^{1}$ Harvard-Smithsonian Center for Astrophysics, Cambridge, MA 02138, USA \\ ${ }^{2}$ University of Amsterdam, Kruislaan 403, 1098 SJ Amsterdam, The Netherlands \\ ${ }^{3}$ SRON, P.O. Box 800, 9700 AV Groningen, The Netherlands \\ ${ }^{4}$ Kapteyn Astronomical Institute, P.O. Box 800, 9700 AV Groningen, The Netherlands
}

\begin{abstract}
We have studied molecular hydrogen emission in a sample of 21 YSOs using spectra obtained with the Infrared Space Observatory (ISO). $\mathrm{H}_{2}$ emission was detected in 12 sources and can be explained as arising in either a shock, caused by the interaction of an outflow from an embedded YSO with the surrounding molecular cloud, or in a PDR surrounding an exposed young earlytype star. The distinction between these two mechanisms can not always be made from the pure rotational $\mathrm{H}_{2}$ lines alone. Other tracers, such as PAH emission or [S I] 25.25 $\mu$ m emission, are needed to identify the $\mathrm{H}_{2}$ heating mechanism. No deviations from a 3:1 ortho/para ratio of $\mathrm{H}_{2}$ were found. Both shocks and PDRs show a warm and a hot component in $\mathrm{H}_{2}$, which we explain as thermal emission from warm molecular gas (warm component), or UV-pumped infrared fluorescence in the case of PDRs and the re-formation of $\mathrm{H}_{2}$ for shocks (hot component).
\end{abstract}

\section{Introduction}

Molecular hydrogen is expected to be ubiquitous in the circumstellar environment of Young Stellar Objects (YSOs). It is the main constituent of the molecular cloud from which the young star has formed and is also expected to be the main component of the circumstellar disk. Most of this material will be at temperatures of 20-30 K and difficult to observe. However, some regions may be heated to temperatures of a few hundred $\mathrm{K}$ and produce observable $\mathrm{H}_{2}$ emission. The intense $\mathrm{UV}$ radiation generated by accretion as well as by the central star itself will create a photodissociation region (PDR), of which the surface layer is heated by collisions with photoelectrically ejected electrons from grain surfaces, in any surrounding neutral material. Another possibility to produce warm $\mathrm{H}_{2}$ is in shocks caused by the interaction of an outflow with the surrounding molecular cloud. Shocks are usually divided into J- or Jump-shocks, and C- or Continuous-shocks. In J-shocks the molecular material is dissociated in the shock front, where the gas is heated to several times $10^{4}$ degrees. Behind the shock front, molecular material will re-form, and warm $\mathrm{H}_{2}$ may be observed in the post-shock gas. C-shocks, in contrast, are not sufficiently powerful to dissociate molecular material, but may produce observable amounts of $\mathrm{H}_{2}$ within the shock front itself.

Until recently the study of $\mathrm{H}_{2}$ in star forming regions has mainly concentrated on the study of the near-infrared ro-vibrational lines observable from the ground. However, the launch of the Infrared Space Observatory (ISO) has opened up the possibility to also study the mid-infrared pure rotational lines of $\mathrm{H}_{2}$, with much lower upper energy levels, and directly detect the thermal emission of warm $\mathrm{H}_{2}$ in a wide variety of sources. In these proceedings we report on our study of $\mathrm{H}_{2}$ lines in the ISO spectra of a sample of 21 YSOs. We will show that emission of warm $\mathrm{H}_{2}$ is common in the environments of intermediate- and high-mass YSOs and can be explained by the phenomena of shocks and PDRs outlined above. 


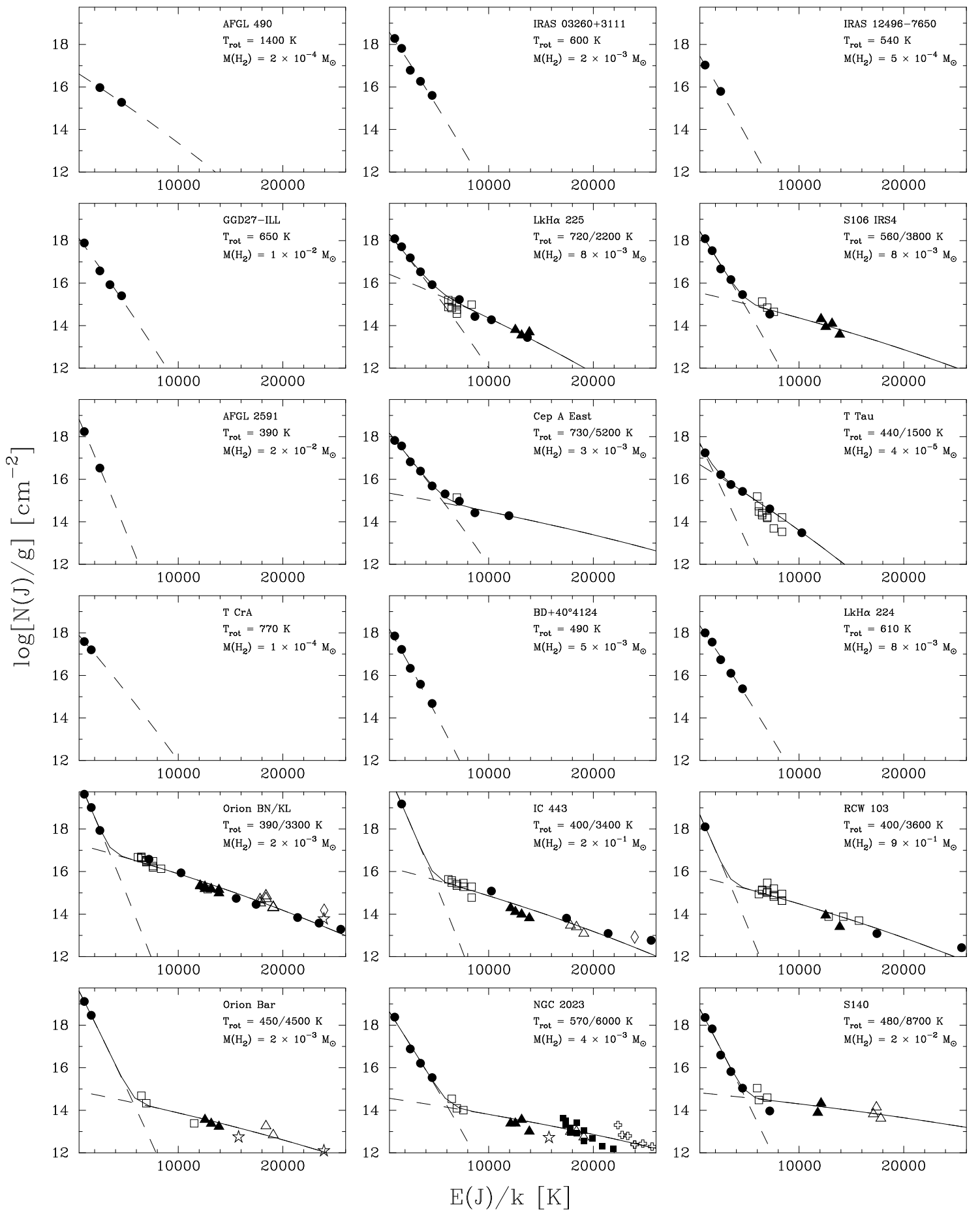

FIGURE 1. $\mathrm{H}_{2}$ excitation diagrams for programme stars (top four rows) and comparison shocks and PDRs (bottom two rows). Shown are apparent columns of $\mathrm{H}_{2}$ in the pure-rotational (0-0; filled dots), 1-0 (open squares), 2-1 (filled triangles), 2-0 (open stars), 3-2 (open triangles), 3-0 (filled squares), 4-3 (open diamonds), and 4-1 transitions (open crosses). Observational errors are smaller than the size of the plot symbol. The Boltzmann distribution fits are plotted as dashed lines. The solid lines show the sum of both thermal components for each source. 

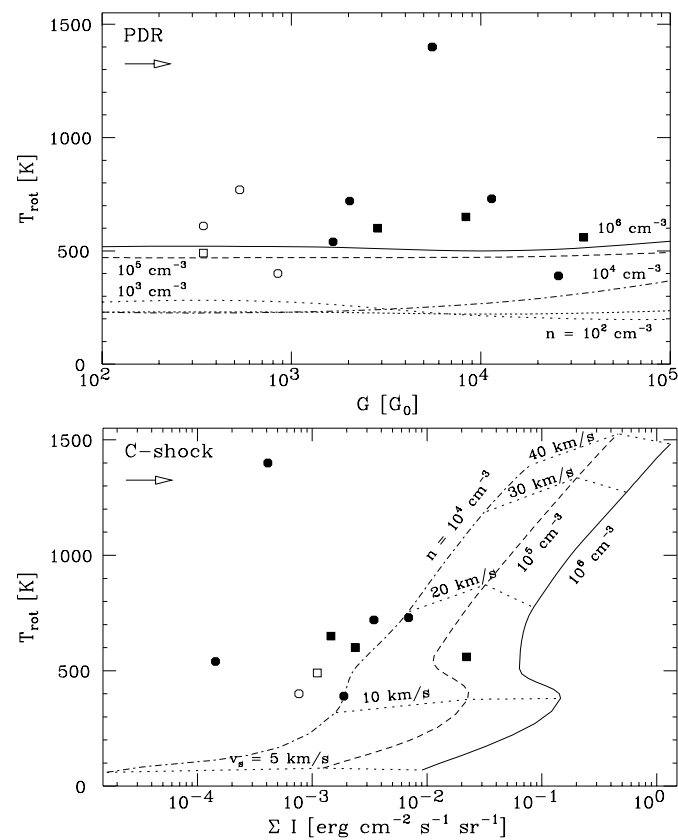

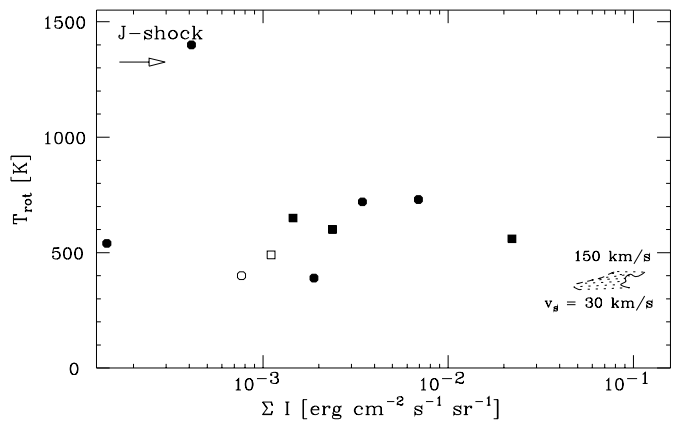

Figure 2. Comparison of observed $\mathrm{H}_{2}$ rotational temperatures to theoretical relation between continuum fluxes and $T_{\text {rot }}$ (PDR models) or summed intensity in all observed lines and $T_{\text {rot }}$ (shocks). Sources which show PAH emission are plotted as squares. Plot symbols are filled for Class I sources. The arrows show the direction of beam dilution.

\section{Observations and Analysis}

ISO Short Wavelength Spectrometer (SWS; $2.4-45 \mu \mathrm{m}$ ) spectra were obtained for a sample of 21 YSOs, mostly of intermediate and high mass. Data were reduced in a standard fashion using calibration files corresponding to OLP version 7.0. In each object, molecular hydrogen line fluxes or upper limits (total flux for line with peak flux $3 \sigma$ ) of $0-0 \mathrm{~S}(0)$ to $\mathrm{S}(11), 1-0 \mathrm{Q}(1)$ to $\mathrm{Q}(6)$ and $1-0 \mathrm{O}(2)$ to $\mathrm{O}(7)$ were determined.

Pure rotational (0-0) $\mathrm{H}_{2}$ emission was detected in 12 out of our 21 sources. Rovibrational (1-0) $\mathrm{H}_{2}$ emission was detected in 4 sources, all of which were also detected in the pure rotational lines. A first inspection of our data shows that $\mathrm{H}_{2}$ emission was only found in the vicinity of early-type $(<\mathrm{B} 4)$ stars or near embedded sources. Qualitatively this is in agreement with one would expect: the strong UV fluxes of early-type stars are expected to produce extended PDRs, whereas embedded YSOs are expected to drive strong outflows, causing a shock as the outflow hits the surrounding molecular cloud.

The $28.2188 \mu \mathrm{m} \mathrm{0-0} \mathrm{S(0)} \mathrm{line} \mathrm{was} \mathrm{not} \mathrm{detected.} \mathrm{This} \mathrm{shows} \mathrm{directly} \mathrm{that} \mathrm{we} \mathrm{did} \mathrm{not}$ detect the cool quiescent $\mathrm{H}_{2}$ in the molecular cloud. A more qualitative analysis of our data can be made by plotting the log of $N(\mathrm{~J}) / \mathrm{g}$, the apparent column density for a given $\mathrm{J}$ upper level divided by the statistical weight, versus the energy of the upper level. For the statistical weight we have assumed the high temperature equilibrium relative abundances of 3:1 for the ortho and para forms of $\mathrm{H}_{2}$. The resulting excitation diagrams are shown in Fig. 1. For comparison we also show excitation diagrams of three sources known to be dominated by shocks (Orion BN/KL peak 1, IC 443 and RCW 103) and three wellknown PDRs (the Orion Bar, NGC 2023 and S140), created using data from literature. In Fig. 1 we also show Boltzmann distribution fits to the low-lying pure rotational lines. The fact that for most sources the the points for ortho and para $\mathrm{H}_{2}$ lie are both well fitted by this nearly straight line proves that our assumption on their relative abundances is correct. For a number of sources, the lines at higher energy levels can be seen to deviate strongly from the Boltzmann fit. In these cases, we have attempted to characterize this behaviour, which may reflect the combined effects of UV-pumped infrared fluorescence and the presence of a very warm, but thin, surface layer in a PDR and may be due to the 
effect of re-formation of $\mathrm{H}_{2}$ in J-shocks, by fitting a second Boltzmann distribution to the higher energy level populations. The resulting excitation temperatures and derived mass of molecular hydrogen are also indicated in Fig. 1.

Employing predictions of $\mathrm{H}_{2}$ emission from PDR, J-shock and C-shock models by Burton et al. (1992), Hollenbach \& McKee (1989) and Kaufman \& Neufeld (1996), we determined the excitation temperature $T_{\text {rot }}$ from the low-lying pure rotational levels as a function of density $n$ and either incident FUV flux $G$ (in units of the average interstellar FUV field $\mathrm{G}_{0}$ ) or shock velocity $v_{s}$ in an identical way as was done for the observations. The results of this procedure, plotted against $G$ (PDRs) or the total flux observed in all lines (shocks) are shown in Fig. 2. Note that there is considerable overlap between the $T_{\text {rot }}$ predicted by PDR, J-shock and C-shock models. This means that pure rotational $\mathrm{H}_{2}$ emission alone cannot distinguish between these mechanisms in all cases and additional information will be needed. However, our ISO spectra provide just such information. Detectable [S I] $25.25 \mu \mathrm{m}$ emission means that a shock must be present, whereas PAH emission is indicative of the presence of a PDR. The presence or absence of ionic lines such as [Si II] $34.82 \mu \mathrm{m}$ can further distinguish between a C-shock and a J-shock. The information from these lines is used in Fig. 2 to distinguish between likely shocks (circles) and PDRs (squares).

In general, the PDR sources fall, within errors, in the parameter space outlined by the PDR models. Since the J-shocks only predict a very narrow range of $T_{\text {rot }}$, only one source, AFGL 2591, is compatible with the observed $\mathrm{H}_{2}$ emission arising in such a dissociative shock. All shock sources are compatible with the range in $T_{\text {rot }}$ predicted by $\mathrm{C}$-shock models. However, the detection of ionic lines in most of these sources shows that a J-shock component must be present. Most likely real astrophysical shocks are never as simple as the purely dissociative or non-dissociative shocks in the employed models, but are made up of a combination of the two, with the non-dissociative component dominating the $\mathrm{H}_{2}$ spectrum.

\section{Conclusions}

We have shown that pure-rotational emission from warm $\mathrm{H}_{2}$ is readily detectable in the vicinity of intermediate- and high-mass YSOs and can be used to gain insight in the physical conditions in the circumstellar material. The main mechanisms that produce warm $\mathrm{H}_{2}$ in these types of environments are shocks and PDRs. No deviations from the $3: 1$ ortho/para ratio of $\mathrm{H}_{2}$ were found for either type of heating mechanism. Both shocks and PDRs show a warm and a hot component in $\mathrm{H}_{2}$. The warm component probes the thermal emission from warm gas. For PDRs the hot component may reflect the combined effects of UV-pumped infrared fluorescence and the presence of a thin, very warm surface layer. In shocks the hot $\mathrm{H}_{2}$ component may be due to the re-formation of $\mathrm{H}_{2}$ with nonzero formation energy. The warm $\mathrm{H}_{2}$ component in shocks appears to be dominated by the non-dissociative part of the shock. The evolution of YSOs is expected to be from

shock-dominated to PDR-dominated and $\mathrm{H}_{2}$ may be one of the best tracers of the end of the outflow phase of a young star.

\section{REFERENCES}

Burton, M.G., Hollenbach, D.J., Tielens, A.G.G.M. 1992, ApJ 399, 563

Hollenbach, D.J., McKee, C.F. 1989, ApJ 342, 306

Hollenbach, D.J., Tielens, A.G.G.M. 1999, Rev. Mod Phys. 71, 173

Kaufman, M.J., Neufeld, D.A. 1996, ApJ 456, 611 